\title{
Imaging of Gasoline-like Sprays with Planar Laser-induced Exciplex Fluorescence using a Stereoscopic Imaging System
}

\author{
Mats Andersson*1, Akichika Yamaguchi², Hua Wang ${ }^{3}$ \\ ${ }^{1}$ Department of Applied Mechanics, Chalmers University of Technology, Göteborg, Sweden \\ ${ }^{2}$ DENSO Corporation, Showa-cho, Kariya-shi, Aichi-ken, Japan \\ ${ }^{3}$ Dantec Dynamics A/S, Skovlunde, Denmark \\ *Corresponding author: f3cma@chalmers.se
}

\begin{abstract}
The role of the fuel injection systems in direct injected gasoline engines is to achieve a suitable fuel vapor distribution, homogeneous or with some degree of stratification, while avoiding unwanted effects such as wall wetting. Planar laser-induced exciplex fluorescence (PLIEF) is a method suitable for the characterization of such sprays since it enables separate imaging of both vapor and liquid phase of fuel simultaneously. In this study a hollow-cone spray generated with an outwards-opening piezo-actuated injector is investigated, with the injector mounted in a constant volume, constant pressure spray chamber with quartz windows, providing a controlled steady test environment. $\mathrm{N}$-hexane is used as surrogate fuel of gasoline, together with exciplex-forming fluorescence tracers - fluorobenzene and diethylmethylamine. Fluorescence excitation is carried out with a parallel laser sheet from the fourth harmonic light of a Nd:YAG-laser $(266 \mathrm{~nm})$ running at $10 \mathrm{~Hz}$. Exciplex fluorescence images from liquid phase and monomer fluorescence spray images from vapor phase can be acquired by a single UV-sensitive CMOS camera equipped with a stereoscope having filters selectively transmitting monomer fluorescence at 295 $\mathrm{nm}$ and exciplex fluorescence at $355 \mathrm{~nm}$. Since the fluorescence is strongly quenched by oxygen, most of the experiments were carried out in a nitrogen atmosphere.

Images were recorded during the injection and at various time steps after the end of the injection, and typical spray development for this type of injector was observed, i.e. the fuel forms an expanding cone, the sheet breaks up to form a vortex structure and the vortices continue to expand after the end of the injection. Fuel vapor is firstly observed at the same locations as the liquid drops, and is then accumulated into the center of the vortices. In addition, penetration of liquid phase and vapor phase are found to be very similar. Various injection pressures have been tested, which shows that increasing the fuel pressure from 10 to $20 \mathrm{MPa}$ results in a larger vortex structure. The fuel evaporation can be followed by studying the evolution of the monomer and exciplex fluorescence as a function of time. At room temperature the vaporization is found to be very slow, but above $40^{\circ} \mathrm{C}$ there is a noticeable presence of vapor at the end of the injection, and at higher temperatures, the vaporization goes even faster.
\end{abstract}

\section{Keywords}

Hollow-cone spray, Exciplex LIF, Phase separation, GDI, LIEF

\section{Introduction}

The role of the fuel injection systems in direct injected gasoline engines is to achieve a suitable fuel vapor distribution, homogeneous or with some degree of stratification, while avoiding unwanted effects such as wall wetting. Therefore it is desirable to characterize the fuel injection systems and how the fuel vaporizes and becomes dispersed in the combustion chamber, in order to investigate and improve the combustion process.

As an extension technique of planar laser-induced fluorescence (LIF), which is proved to be a powerful imaging technique to visualize distributions of fuel, air or even combustion species, planar laser-induced exciplex fluorescence (PLIEF) provides spectrally separated fluorescence from the liquid and vapor phases and therefore is a novel and significant advance for imaging and quantifying vaporizing sprays [1,2]. In this method, the fuel is doped with two different tracers. The two tracers are selected so that one, or both, of the tracers are excited by the incoming laser light and upon excitation forming an excited state complex, an exciplex, with the other tracer molecule. The fluorescence of this exciplex is red-shifted compared to the fluorescence of the individual molecules, the monomers. The exciplex formation is efficient only in liquid phase where the tracers are in closer proximity and the solvent can act stabilizing for the complex. One of the advantages with LIEF as compared with LIF is that it enables imaging of the vapor phase without interference from the liquid phase.

This technique has been used for visualization $[3,4]$ and quantitative measurements with both diesel-like $[5,6]$ and gasoline-like tracer-fuel mixers [7,8]. For most of the studies in the literature, the fluorescence from the spray was 
imaged using two cameras, equipped with filters transmitting wavelengths corresponding to monomer and exciplex emission, respectively. These two images ideally correspond to the fuel distribution in vapor and liquid phase.

In this study, a stereoscopic imaging system is used to image both monomer and exciplex fluorescence from a gasoline-like spray, which generates a hollow-cone spray. The imaging system includes a stereoscope and therefore only one camera is needed. Spray development of the vapor and liquid phase with time after start of injection (asoi) is well captured by this imaging system. In addition, influences from injection pressure and ambient conditions have also been observed and analyzed to investigate how these factors influence the location and relative abundance of liquid and vapor phase fuel.

\section{Experiment setup}

Spray development was investigated in a constant volume/constant pressure spray chamber, shown in Figure 1. The chamber can provide a constant air flow, in order to ventilate the injected fuel; however, the air velocity is significantly lower than the spray velocity and therefore it has negligible influence on the spray development. The chamber is equipped with three quartz windows during the study, providing good optical access for both laser light sheet and fluorescence signals.
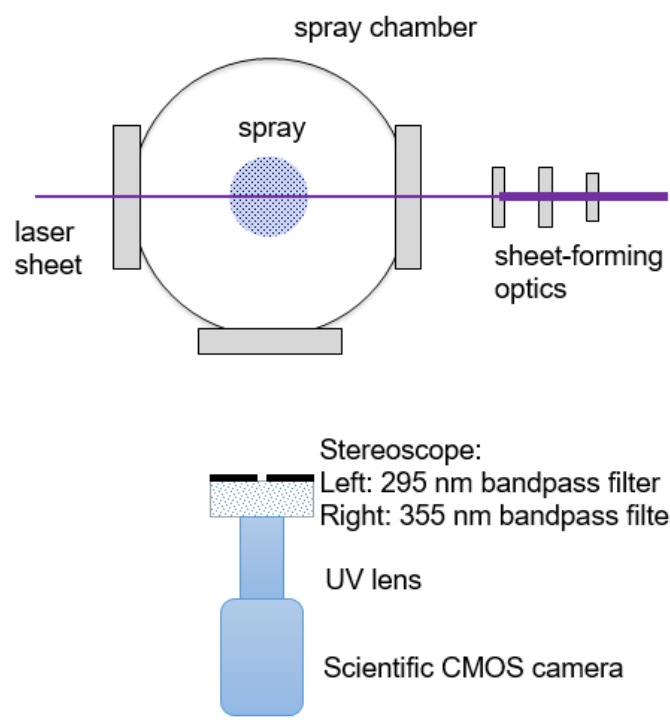

Figure 1. Schematic of the experimental setup as seen from above.

An outward opening piezo actuated GDI injector (Bosch type) was used during the experiments and mounted at the top of the spray chamber. The outward opening needle creates a hollow cone spray shape with vorticies [9-13]. The injector driver supplies a certain voltage over the piezo stack, which expands rapidly and acts on the needle. The needle lift will increase with increased voltage. Due to the fast opening and closing of the needle, the injector can be opened and closed multiple times during one engine cycle and this in addition to the possibility to use varying needle lifts provide many degrees of freedom for spray control but is also a challenge for engine calibration. The injector type is used up to 200 bar fuel injection pressure and provides great atomisation, and limited vertical penetration.

The fuel temperature was kept constant by regular fuel injections. The chamber gas temperature was constantly monitored by thermocouples during all measurements. The fuel pressure is well controlled by a pressurized air driven Haskel pump connected to a pressure accumulator. The nominal electrical control pulse was $0.4 \mathrm{~ms}$, but due to the ramping of the control voltage for opening and closing, the total time during which fuel was injected was observed to be $\sim 0.8 \mathrm{~ms}$.

$\mathrm{N}$-hexane was used as surrogate fuel of gasoline, together with exciplex fluorescence-forming tracers fluorobenzene (FBZ) and diethylmethylamine (DEMA). This is a well-characterized tracer combination [14] with minimal spectral cross-talk and good co-evaporation properties, and has been applied in several studies [15-18]. The concentrations of tracers used in this work was $3 \% \mathrm{FBZ}$ and $7 \%$ DEMA by volume.

Fluorescence excitation was carried out with a parallel laser sheet from the fourth harmonic light of a Nd:YAG-laser $(266 \mathrm{~nm}$ ) running at $10 \mathrm{~Hz}$, and the energy of each pulse was around $20 \mathrm{~mJ}$. The laser light was formed into a 50 
$\mathrm{mm}$ high sheet passing just under the injector. Exciplex fluorescence image from liquid phase and monomer fluorescence spray image from vapor phase was acquired by a single UV-sensitive scientific CMOS camera equipped with a stereoscope having filters selectively transmitting monomer fluorescence at $295 \mathrm{~nm}$ and exciplex fluorescence at $355 \mathrm{~nm}$, a simpler system than the 4 way-mirror arrangement reported for fuel and temperature imaging [19]. The image recording rate was the same as the fuel injection rate, $1 \mathrm{~Hz}$. Since the fluorescence is strongly quenched by oxygen, most of the experiments were carried out in a nitrogen atmosphere.

The influence from variant injection pressure (100 or 200 bar fuel pressure) and ambient temperature (21, 39, 56 or $68^{\circ} \mathrm{C}$ ) has been investigated in this study. For all tested conditions, images were recorded at various time steps during and after the end of the injection and consequently the spray development of both vapor and liquid phase can be observed and analyzed.

\section{Results and discussion}

Images of the spray have been captured with the spray chamber pressurized with air or with nitrogen to assess the influence of quenching by oxygen molecules on the fluorescence yield. Figure 2 shows exciplex-monomer image pairs, recorded at the same conditions except the spray chamber being fed by air or nitrogen. In addition to the images, horizontal intensity profiles calculated at the centre of the two images are shown. The two exciplex images are very similar with vortices having a low drop density at the middle, the main difference being a lower signal intensity of about a factor of 3 in air. The monomer image recorded with a nitrogen atmosphere shows as expected a vapor fuel distribution significantly different from that of the liquid drops (exciplex), whereas the monomer image recorded in air has a very low signal intensity, more than 50 times lower than in nitrogen. The character of the monomer images is also slightly different, likely due to the strong quenching of the vapor monomer signal resulting in monomer fluorescence from the liquid phase contributing to the detected monomer fluorescence. A monomer fluorescence image in air with a good signal intensity could be obtained using an image intensifier in front of the camera. However, for this study of spray properties analysing liquid and vapour fuel distribution all further imaging was carried out in nitrogen atmosphere since this provided good quality monomer and exciplex images of comparable intensity using the stereoscopic imaging system without image intensifier.
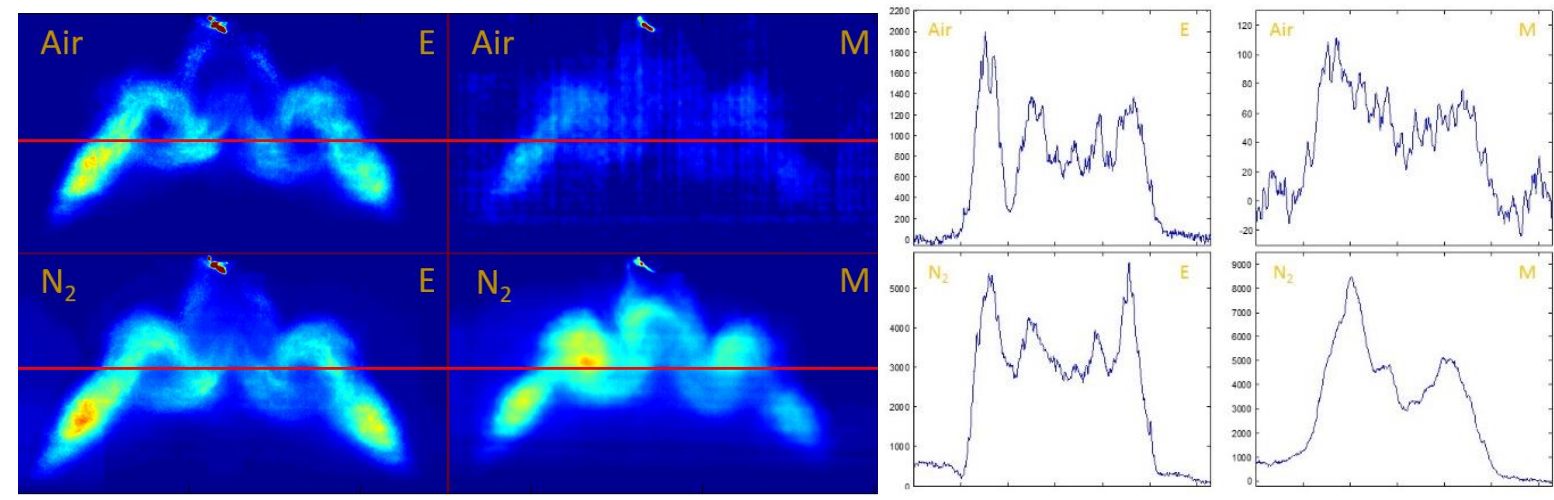

Figure 2. Left: averaged exciplex (left column) and monomer (right column) images recorded in air (upper row) and nitrogen (lower row) at 4.2 bar pressure and $56^{\circ} \mathrm{C}$ gas temperature, $3 \mathrm{~ms}$ asoi with a fuel pressure of 100 bar. Image size $95 \times 53 \mathrm{~mm}$. Right: Intensity profiles taken through the middle of each image of Left side (labeld as red line).

In each measurement point (pressure, temperature and time asoi) 20 images were recorded to obtain reasonable statistics, and from the 20 images average images were calculated. In Figure 3 averaged images at some selected measurement points are shown together with two of the individual image pairs. In the further description of the results averaged images will be displayed since general trends are more easily identified in these and the arbitrariness in the selection of one individual image can be avoided. On the other hand there are important features, which only can be seen in the individual images that will be commented on in the further analysis. Naturally the structures in the averaged images appear more smeared out, whereas the liquid structures, especially at later times appear as drops concentrated to streaks in the individual images. The vapour clouds are more homogeneous, although with internal concentration differences, and have sharp boundaries. 


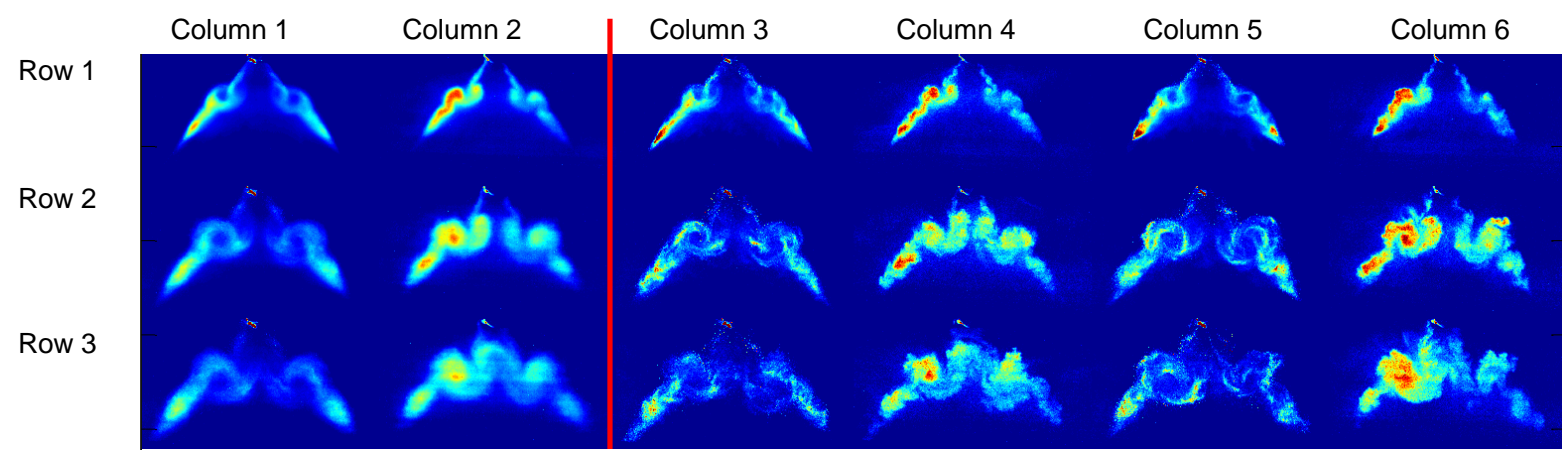

Figure 3. Averaged (Column 1 \& 2) and individual (Columen 3- 6) image pairs, where left image in each pair (columns 1, 3 and 5 ) is an exciplex image and the right image (columns 2, 4 and 6) is a monomer image. The images are recorded at 4.2 bar nitrogen pressure and $56^{\circ} \mathrm{C}$ temperature, a fuel pressure of 100 bar and 1 (row 1 ) 2 (row 2) and 3 (row 3 ) ms asoi. Image size $95 \times 53 \mathrm{~mm}$.

An overview of the spray development with time can be followed by a series of exciplex images displayed in Figure 4. The images, in particular the ones recorded at the earlier time steps, show a non-symmetric intensity where the left part of the spray is much more intense. The reason for this is that the laser light enters from the left and due to the high drop density the light is strongly attenuated and only a fraction of the incident light continues to the right part of the spray. Another effect of the high drop density is multiple light scattering resulting in light being scattered by drops outside of the laser sheet, and some intensity appears to be generated inside the cone.

Initially the cone develops rapidly during the injection, and soon there is a perturbation about $20-25 \mathrm{~mm}$ from the injector. This perturbation leads to the formation of the vortex structure, with two counter-rotating vortices, a larger outer vortex and a smaller inner one. The vortices continue to grow also long after the end of injection. At the bottom of the spray, i.e. downstream of the point where the vortices are formed, the cone structure is preserved without large movements the fuel. Close to the injector traces of the cone can still be observed, along with scattered larger drops (seen in individual images).

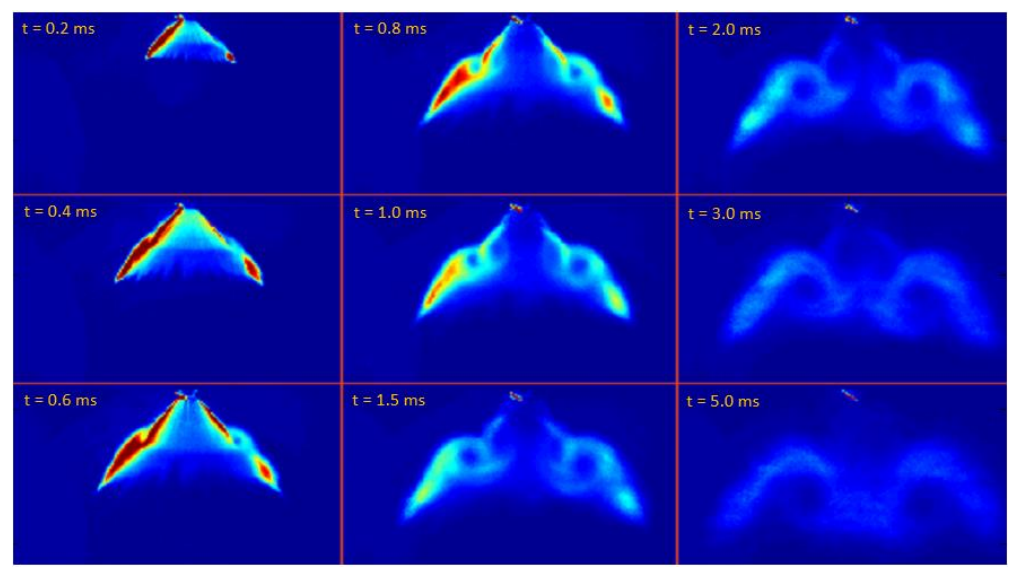

Figure 4. A series of exciplex fluorescence images recorded at 4.2 bar nitrogen pressure and $56^{\circ} \mathrm{C}$ temperature, a fuel pressure of 100 bar. The images are recorded at 0.2, 0.4, 0.6 (left column), 0.8, 1.0, 1.5 (middle column), 2.0, 3.0, 5.0 ms asoi (right column).

To analyse the fuel evaporation, both monomer fluorescence and exciplex fluorescence images at different time after start of injection are shown in the Figure 5, under four different ambient temperatures. At all the temperatures tested, fuel vapor is firstly observed at the same locations as the liquid drops, and then it is accumulated into the center of the vortices, which can be well observed in all exciplex images from shortly after start of injection (1ms). In the exciplex images there is a local minimum in the center of the votices, whereas there is a local maximum at the same position, which is in agreement with earlier observations using a combination of planar LIF and Mie scattering [20]. In addition, penetration of liquid phase and vapor phase are found to be very similar.

The fuel evaporation can be followed by studying the evolution of the monomer and exciplex fluorescence as a function of time. At room temperature $\left(21^{\circ} \mathrm{C}\right)$ the vaporization is found to be very slow, and consequently the 
exciplex signal (corresponding to the liquid phase) is much stronger than the monomer signal (corresponding to the vapor phase). When the ambient temperature is increased to around $40^{\circ} \mathrm{C}$, vaporization of fuel is more observed, particularly after end of injection. Correspondingly, the intensity value of exciplex fluorescence images is slightly reduced after end of injection, proving more fuel was vaporized. It can also be noted the smaller inner vortex, which is barely seen in the exciplex images, appears more clearly in the monomer images and this fuel vapor moves towards the center of the cone to fill the volume about $20 \mathrm{~mm}$ below the injector.

When in-chamber temperature is above $40^{\circ} \mathrm{C}$ there is a noticeable presence of vapor at the end of the injection. At this temperature, monomer fluorescence images generally show a higher intensity value in the spray region, compared with exciplex fluorescence images. At higher temperatures, the vaporization goes even faster. Nonetheless, there are still some big drops remaining in the spray a relatively long time after end of injection at the higher temperatures. The influence of temperature on the appearance of vortices seems to be small, but their size is smaller at later times at higher temperature, likely due to a loss of momentum as the drops vaporize. Increasing of temperature leads to more vapor and less liquid in the vortices region.
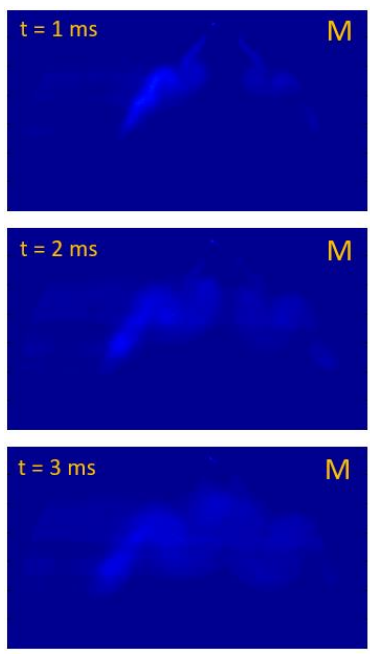

(a) Ambient temperature $21^{\circ} \mathrm{C}$
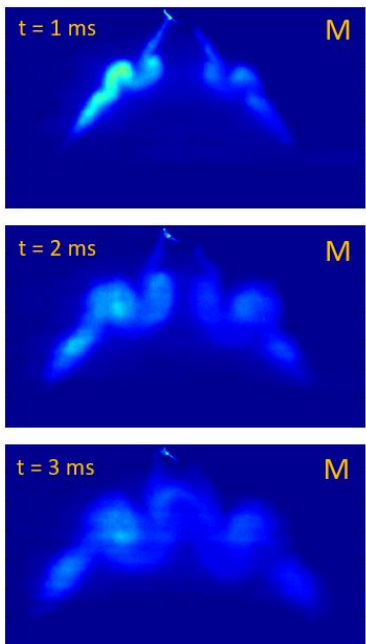

(c) Ambient temperature at $56^{\circ} \mathrm{C}$
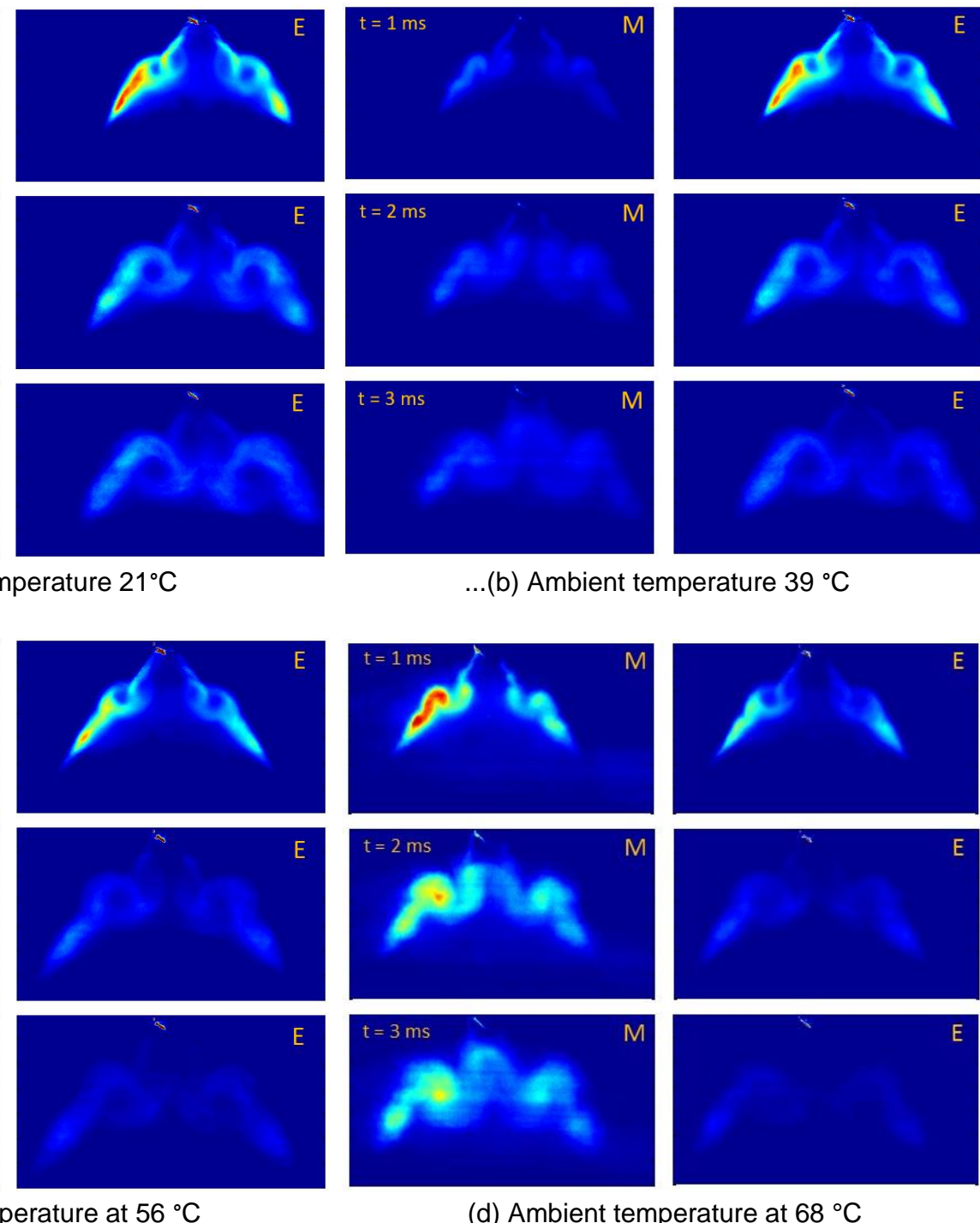

(d) Ambient temperature at $68^{\circ} \mathrm{C}$

Figure 5. Monomer fluorescence $(\mathrm{M})$ and exciplex fluorescence $(\mathrm{E})$ images at different time after start of injection under different ambient temperature, 4.2 bar nitrogen pressure, 100 bar injection pressure

The influence of fuel pressure was investigated by performing measurements at 100 and 200 bar. Figure 6 shows a two sets of images (temperature 39 and $56^{\circ} \mathrm{C}$ ) recorded with a fuel pressure of 200 bar, but otherwise the same conditions as in the corresponding images shown in Figure 5. It is clear that the higher fuel pressure leads to a much stronger vortex formation, with larger vortices at each of the time steps. It should in particular be noted that 
much of the fuel remaining at the bottom of the cone at 100 bar, is dragged into the vortices at a fuel pressure of 200 bar. Furthermore, it can be noted that the intensity ratio between monomer and exciplex fluorescence is higher at 200 bar compared to 100 bar, which shows that vaporization is faster at the higher fuel pressure.

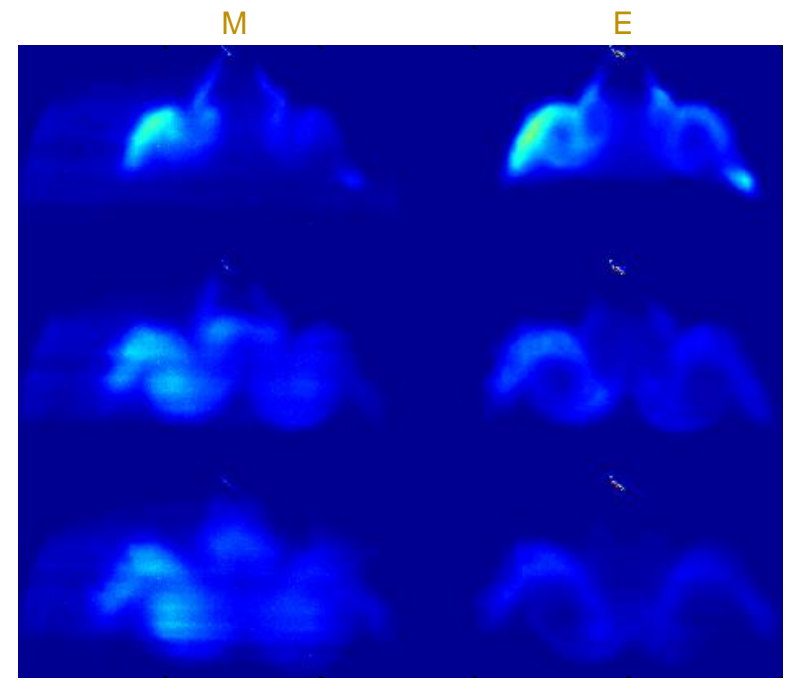

Left panel: $\mathrm{T}=39^{\circ} \mathrm{C}$

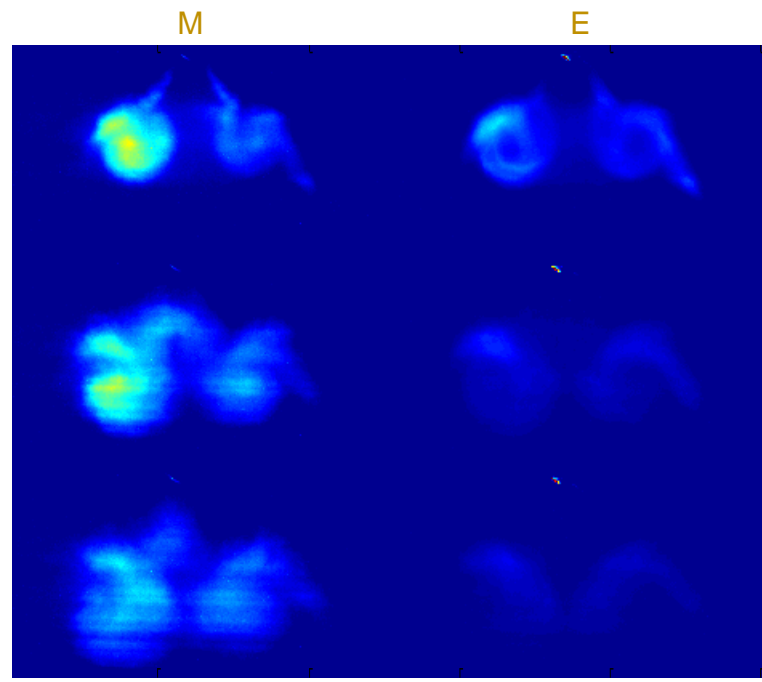

Right panel: $\mathrm{T}=56^{\circ} \mathrm{C}$

Figure 6. Averaged monomer fluorescence (left) and exciplex fluorescence (right) image pairs recorded at chamber temperature $39^{\circ} \mathrm{C}$ (left panel) and $56^{\circ} \mathrm{C}$ (right panel), pressure 4.2 bar and fuel pressure 200 bar, at various times asoi:

$1 \mathrm{~ms}$ top row, $2 \mathrm{~ms}$ middle row and $3 \mathrm{~ms}$ bottom row.

The variations in fuel distribution from injection to injection was analyzed by calculating images representing the standard deviation and coefficient of variation (standard deviation divided by the average). Figure 7 shows a representative example of standard deviation and coefficient of variation images recorded at $T=56^{\circ} \mathrm{C}$ and $P_{\text {fuel }}=$ 100 bar together with the averaged image. In the standard deviation images it can be seen that the largest values are obtained where there is a high fuel concentration. This shows that even if the major part of the fuel is concentrated to certain areas, there are still significant fluctuations between injections of the actual distribution. This is also what can be seen in individual images as the ones shown in Figure 3. The strongest relative variations in fuel concentrations as seen in the coefficient of variation images are found along the borders of the spray and particularly on the outside of the cone. This is an effect of a high-concentration fuel cloud with a sharp edge, but the edge appearing at slightly different locations in each injection. In the coefficient of variation images there are dots appearing in the area close to the nozzle. This is due to larger fuel drops, likely generated during the injector closing event, still remaining in the area around the injector.

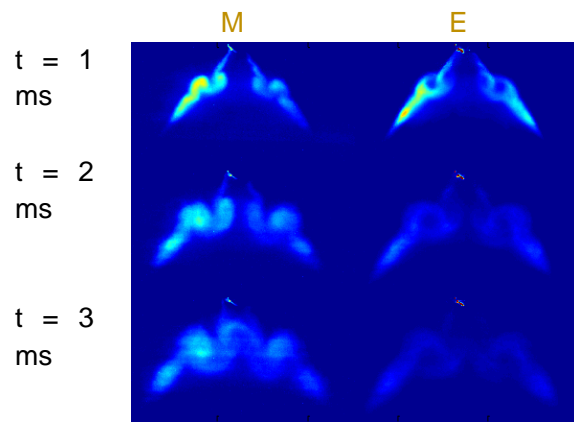

averaged images

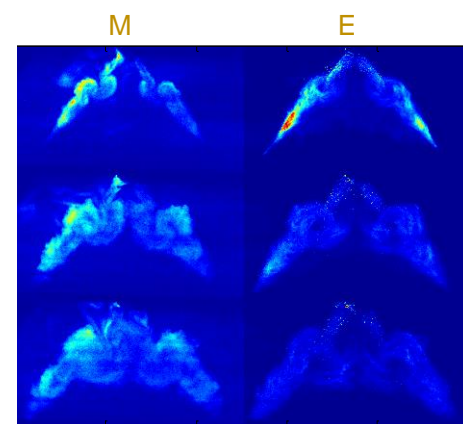

standard-deviation images

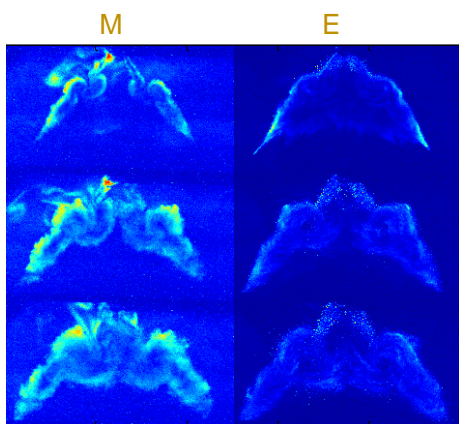

coefficient-of-variation images

Figure 7. Averaged images (left), standard-deviation images (middle) and coefficient-of-variation images (right). In each panel monomer image is in the left column and the exciplex image in the right column. The images are recorded at 4.2 bar nitrogen pressure and $56^{\circ} \mathrm{C}$ temperature, a fuel pressure of 100 bar and 1 (top row) 2 (middle row) and 3 (bottom row) ms asoi. Image size $95 \times 53 \mathrm{~mm}$. 


\section{Conclusions}

Fuel sprays from a piezo-actuated outwards-opening gasoline injector were investigated using the planar laserinduced exciplex fluorescence technique. In this application a single CMOS camera without intensifier was used with a stereoscope. With appropriate filters, images of monomer and exciplex fluorescence with good signal strengths could be obtained. The distribution of fuel in liquid and vapor phase was imaged at various times after start of injection, at different gas temperatures in the spray chamber and with different fuel injection pressures. It was found that the double vortex structure, characteristic for this type of injector was found, and that the drops predominantly remain at the outer part of the vortices, whereas the fuel vapor moves towards the center of the vortices, but also towards the center of the cone. With the $n$-hexane-based fuel, there is a significant difference in the observed fractions of fuel in liquid and vapor phase when temperature is varied in the $20-70^{\circ} \mathrm{C}$ range. When the fuel injection pressure is increased from 100 to 200 bar, the vortices become significantly larger and also the evaporation becomes faster.

\section{Acknowledgements}

Financial support was obtained from the Combustion Engine Research Centre (CERC) at Chalmers, and the Swedish Energy Agency. The imaging system was provided by Dantec Dynamics. Stimulating discussions with Petter Dahlander are gratefully acknowledged.

\section{References}

[1] Melton, L.A., 1983, Applied Optics, 22, 2224-2226.

[2] Fansler, T. and Parrish, S., 2015, Measurement Science and Technology, 26, 012002(34pp).

[3] Ghandhi, J. B., Felton, P. G., Gajdeczko, B. F., and Bracco, F. V., 1994, SAE Paper 940394.

[4] Fujimoto, H., Choi, D., Shima, Y., and Senda, J., 2002, Measurement Science and Technology, 13, pp. 391

[5] Payri, F., Pastor, J.V., Pastor, J.M. and Juliá, J.E., 2006, International Journal of Engine Research, 7, pp. 7789

[6] Rogler, P., Grzeszik, R., Arndt, S. and Aigner, M., 2007, SAE Paper 2007-01-1827

[7] Chen, H., Xu, M., Zhang, G., Zhang, M. and Zhang, Y., 2010, ASME Conf. Proc. 2010 (49446) 391-403

[8] Froba, A., P., Rabenstein, F., Munch, K., U., and Leipertz, A., 1998, Combust. Flame, 112, pp. 199-209.

[9] Skogsberg, M., Dahlander, P., Denbratt, I., 2007, SAE Technical Paper, 2007-01-1409.

[10] Baecker, H., Kaufmann, A., Tichy, M., 2007, SAE Technical Paper, 2007-01-1407.

[11] Hemdal, S., Denbratt, I., Dahlander, P., Wärnberg, J., 2009, SAE Technical Paper, 2009-01-1496.

[12] Zigan, L., Schmitz, I., Flügel, A., Wensing, M., Leipertz, A., 2011, Fuel, 90, 348-363.

[13] Schmid, A., Schneider, B., Boulouchos, K., Wigley, G., 2014, Atomization and Sprays, 24, 859-873.

[14] Düwel, I., Koban, W., Zimmermann, F.P., Dreier, T., Schulz, C., 2009, Applied Physics B, 97, pp 909-918.

[15] Fansler, T.D., Drake, M.C., Gajdeczko, B. Düwel, I., Koban, W., Zimmermann, F.P., Schulz, C., 2009, Measurement Science and Technology, 20, 125401.

[16] Zhang, G.M., Xu, M., Zhang, Y.Y., Zhang, M., Cleary, D.J., 2012, Atomization and Sprays, 22, pp 861-878.

[17] Zeng, W., Xu, M., Zhang, Y., Wang, Z., 2013, Proceedings of the Combustion Institute, 34, pp 1677-1685.

[18] Shim, Y.S., Choi, G.M., Kim, D.J., 2009, International Journal of Multiphase Flow, 35, pp 885-895.

[19] Wieske,P., Wissel, S., Grünefeld, G., Pischinger, S., 2006, Applied Physics B, 97, pp 323-329.

[20] Andersson, M., Wärnberg, J., Hemdal, S., Dahlander, P., Denbratt, I., SAE Technical Paper 2011-01-1889. 